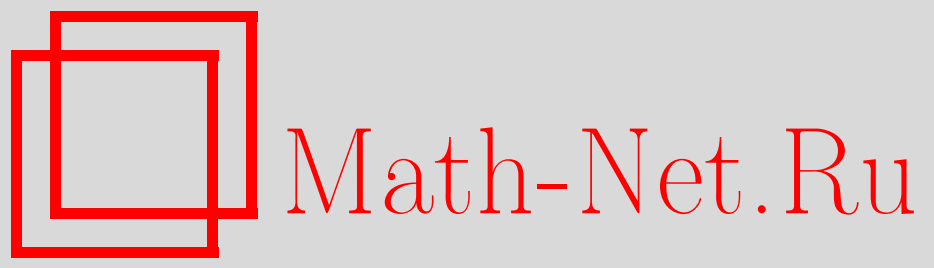

К. Олещко, А. Ю. Хренников, О применениях $p$-адики в геофизике: линейная и квазилинейная диффузия водомасляной эмульсии, ТМФ, 2017, том 190, номер 1, 179 190

DOI: https://doi.org/10.4213/tmf9142

Использование Общероссийского математического портала Math-Net.Ru подразумевает, что вы прочитали и согласны с пользовательским соглашением http://www.mathnet.ru/rus/agreement

Параметры загрузки:

IP: 54.237 .206 .68

26 апреля 2023 г., 16:29:29

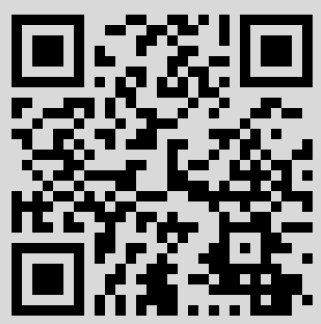




\title{
О ПРИМЕНЕНИЯХ $p$-АДИКИ В ГЕОФИЗИКЕ: ЛИНЕЙНАЯ И КВАЗИЛИНЕЙНАЯ ДИФФУЗИЯ ВОДОМАСЛЯНОЙ ЭМУЛЬСИИ
}

\begin{abstract}
Обсуждаются в весьма общей постановке возможности применения $p$-адики в геофизике с использованием $p$-адического диффузионного представления основных уравнений для динамики жидкости в капиллярах в пористых средах и сформулировано несколько математических проблем, мотивированных этими применениями. Подчеркивается роль $p$-адических всплесков как мощного средства получения аналитических решений диффузионных уравнений. Так как p-адическая диффузия является особым случаем фрактальной диффузии, а последняя тесно связана с фрактальной структурой конфигурационного пространства, p-адическая геофизика может рассматриваться как новый подход к фрактальному моделированию геофизических процессов.
\end{abstract}

Ключевые слова: основное уравнение, геофизика, водомасляная эмульсия, $p$-адические числа, $p$-адическая диффузия, квазилинейная $p$-адическая диффузия.

DOI: $10.4213 / \operatorname{tmf} 9142$

\section{1. ВВЕДЕНИЕ}

В течение последних 15 лет $p$-адика активно использовалась в исследовании различных проблем теоретической физики (в теории струн, космологии, квантовой теории, неупорядочных системах (спиновых стеклах)) и биологии (в моделировании процесса мышления и в генетике). Недавно $p$-адические методы (в основном теория $p$-адических всплесков [1]-[4]) и особенно теория $p$-адических всплесков начали применяться в геофизике, например при моделировании диффузии в случайных

Статья написана при финансовой поддержке грантов SENER-CONACYTHidrocarburos, Yacimiento petrolero como un reactor fractal, № 168638 и Consejo Nacional de Ciencia y Tecnología (CONACYT), Mexico (грант 312-2015), Fronteras de la Ciencia.

${ }^{*}$ Centro de Geociencias, Universidad Nacional Autónoma de México (UNAM), Campus UNAM Juriquilla, Querétaro, México

${ }^{\dagger}$ International Center for Mathematical Modelling in Physics and Cognitive Sciences, Mathematical Institute, Linnaeus University, Växjö, Sweden.

E-mail: andrei.khrennikov@lnu.se 
пористых средах: динамики жидкости (масла, воды, водомасляной эмульсии [5]) в сложной сетке пор с известной топологией.

Основная мотивация использования $p$-адики в геофизике заключается в древоподобной структуре сети капилляров в случайных пористых средах. Жидкости текут сквозь эти древоподобные сети из одного капилляра в другой. Следовательно, может быть полезно моделировать их динамику, представив конфигурационное пространство в виде сети капилляров, т.е. работать не в евклидовом физическом пространстве, где находится пористая среда и которое моделируется с помощью вещественных чисел, а в древоподобном пространстве капилляров, которое может моделироваться как $p$-адическое (или ультраметрическое в более общем смысле) пространство.

Начиная с фундаментальных работ Воловича [6], [7], посвященных $p$-адическим струнам, p-адические методы широко распространились в теоретической физике: в теории струн, космологии, квантовой теории, в неупорядоченных системах - спиновых стеклах (см., например, [6]-[17] и ссылки в этих работах). Некоторые авторы, стимулированные развитием $p$-адической теоретической физики, использовали $p$-адику в исследовании различных проблем биологии, например при моделировании процесса мышления [18].

Здесь уместно сделать замечание о различных способах применения $p$-адики и в различных приложениях к физике и биологии. В фундаментальной физике, теории струн и квантовой теории $p$-адика применялась благодаря ее особенным свойствам, связанным с теорией чисел. Эта исследовательская программа может быть названа "от теории чисел к физике" [9]. А вот в теории неупорядоченных систем изучалась ультраметричность, т. е. иерархическая структура матрицы Паризи может быть выражена с помощью р-адики [12], [13]. Эта исследовательская программа может быть названа "от ультраметрического представления иерархии к физике неупорядоченных систем". В общем случае эта программа реализуется в ультраметрическом представлении иерархии сложных энергетических ландшафтов [13]-[15]. Такой же подход был использован в моделировании процесса мышления [18]: иерархия в ментальной информации была выражена с помощью ультраметрических древоподобных (например, $p$-адических) структур. Однако, в отличие от спиновых стекол, укладки белка или генетики, в моделировании мышления это ментальное ультраметрическое представление основывается на так называемых "физических деревьях", деревьях нейронных сетей. Подход с "физическими деревьями" также используется в геофизических применениях. Здесь можно найти много древоподобных структур, от картин эрозии до сетей капилляров в случайных пористых средах. Такие физические древоподобные структуры могут быть представлены как ультраметрические, в частности р-адические, пространства [5].

В случае динамики частиц в таких физических древоподобных структурах мы можем изучать ультраметрические основные уравнения так же, как при моделировании кинетики сложных энергетических ландшафтов. Таким образом, в качестве первого шага может стать изучение моделей $p$-адической диффузии, используемых в работах [14], [15] (с использованием всплесков, как в работах [2]-[4]). Заметим, что уравнение $p$-адической диффузии является специальным математическим представлением аномальной диффузии ${ }^{1)}$. Наша $p$-адическая модель может

\footnotetext{
1) В настоящее время существуют многочисленные теоретические и экспериментальные подтверждения того, что диффузионная модель для неупорядоченных систем отличается от классического описания Фика и является аномальной, т. е. дисперсия соответствующего стохастического процесса нелинейно зависит от времени (см., например, [19]).
} 
рассматриваться как обобщение закона диффузии Фика для евклидовых решеток на случай $p$-адических древоподобных сетей капилляров (для сравнения см. [20], см. также [21], [22]).

Более детальный анализ приводит к некоторым обобщениям, которые мы планируем обсудить в этой статье. Во-первых, применение закона Аррениуса для вычисления вероятностей переходов, который активно изучается в динамике на сложных энергетических ландшафтах и выражает особую взаимосвязь между энергетическим потенциалом $U(x)$ и вероятностями переходов, не вполне обосновано в геофизических применениях. Мы должны продолжить нашу работу с общими вероятностями переходов (в духе исследований в работах [3], [4], [23]-[26]). В геофизике мы должны моделировать динамику баланса для некоторых типов жидкостей, например водомасляной эмульсии. Более того, важно делать различие между жидкостью, которая не связана с поверхностью капилляров (например, свободная нефть или вода), и той ее частью, которая связана с поверхность капилляров (например, система "твердое тело + связанная нефть"). Такая многокомпонентная динамика представлена системой основных уравнений диффузионного типа. Нас интересует поведение решений таких систем.

Заметим, что дифференциальные операторы $D^{\alpha}$, определяющие особый (но очень важный) класс $p$-адических уравнений диффузии (в частности, оператор Владимирова [9]), являются $p$-адическим аналогом дробного дифференцирования. Эти операторы связаны с древовидной структурой конфигурационного пространства, в нашем случае с сеткой капилляров в пористых средах. Эта особенность дробных дифференциальных операторов активно изучалась в стандартных применениях вещественного анализа в геофизике. Здесь дробные уравнения реакции-диффузии играют важную роль. Интересные модели, основанные на численном моделировании для дробной диффузии, были предложены и изучались в работах [27]-[33].

$p$-Адический диффузионный подход имеет некоторые преимущества. Одно из них чисто теоретическое: моделирование на "древоподобном конфигурационном пространстве" (на сетках капилляров). Этот подход может привести к новым особенностям динамики, которые не заметны в вещественном аналитическом подходе с дробными дифференциальными операторами. Однако основным преимуществом является возможность найти аналитические решения дробных диффузионных уравнений с помощью теории $p$-адических всплесков [1]-[5], [34]. В данном случае ключевой момент заключается в том, что $p$-адические всплески являются собственными функциями $p$-адических дробных дифференциальных операторов. Это дает возможность найти явные решения, например, задачи Коши, в виде ряда всплесков. В то же время вещественный подход к дробной диффузии обычно основывается на численных методах, так как найти аналитические решения не просто (если вообе возможно).

Практически все исследования $p$-адической диффузии относятся к линейной динамике. Мы можем обратить внимание только на исследование квазилинейной p-адической диффузии (раздел 6, подробнее см. [34]; особый класс нелинейных стационарных уравнений также изучался в работе [24]). Нелинейные $p$-адические основные уравнения возникают естественным образом в случае таких физических процессов, в которых вероятности переходов зависят от плотности жидкости. Следовательно, их изучение является важным для применений, в частности в геофизике. В этой статье эту проблему мы сводим к краткому, но, как мы надеемся, стимулирующему изложению в разделе 6. 
Наконец, заметим, что дробное дифференцирование связано с фрактальным анализом. Последний интенсивно применялся в геофизике, в частности одним из соавторов этой статьи [35], [36]. Следовательно, использование $p$-адической модели может рассматриваться как продолжение фрактального подхода к моделированию случайных пористых сред. В этом подходе представлено обобщение диффузионного закона Фика для евклидовых решеток на случай фрактальных решеток (см. [20]). В дискуссионном разделе указанной статьи было сделано ударение на следующем: “для самоподобных фракталов мы ожидаем, что плотность $p(r, t)$ имеет разрывы на всех шкалах длин. Несмотря на эти разрывы, мы можем очень хорошо аппроксимировать $p(r, t)$ огибающей функиией, дающей решение уравнения диффузии; это является, по нашему мнению, обобщением диффузии Фика на случай фрактальных решеток". В настоящей статье мы рассматриваем обобщение диффузии Фика на случай $p$-адических древоподобных трехмерных сетей капилляров, наполненных водомасляной эмульсией, - прототипов сложных пористо-жидкостных систем.

\section{2. p-АДИЧЕСКОЕ ПРЕОБРАЗОВАНИЕ ФУРЬЕ И ДРОБНЫЕ ПСЕВДОДИФФЕРЕНЦИАЛЬНЫЕ ОПЕРАТОРЫ}

Представим кратко основы $p$-адического преобразования Фурье и введем $p$-адические дробные дифференциальные операторы (подробнее см., например, [3]).

Напомним, что элементы поля $p$-адических чисел $\mathbb{Q}_{p}$ могут быть выражены в виде следующего ряда (который сходится относительно метрики, определенной с помощью $р$-адической оценки):

$$
x=\sum_{j=m}^{\infty} \alpha_{j} p^{j},
$$

где $m=0, \pm 1, \pm 2, \ldots$ и $\alpha_{j}=0,1, \ldots, p-1$. В этом представлении мы полагаем, что $\alpha_{m} \neq 0$. Для ненулевого $x$, задаваемого рядом $(1), p$-адическая норма (аналог абсолютного значения) определяется как $|x|_{p}=p^{-m}$ и по определению $|0|_{p}=0$.

Чтобы ввести характеры на поле $\mathbb{Q}_{p}$ (которое является аддитивной локально-компактной группой), мы должны ввести дробную часть $\{x\}_{p}$ от $x$ : это блок ряда (1), соответствующий отрицательным степеням $p$.

$p$-Адический шар радиуса $R$, где $R$ - некоторая степень $p$, с центром в точке $a \in \mathbb{Q}_{p}$ определяется как

$$
B_{R}(a)=\left\{x \in \mathbb{Q}_{p}:|x-a|_{p} \leqslant R\right\} .
$$

Заметим, что $\mathbb{Q}_{p}$ может рассматриваться как метрическое пространство с метрикой $d_{p}(x, y)=|x-y|_{p}$. Оно полно и локально-компактно, так как любой шар $B_{R}(a)$ является компактным. Эта метрика является так называемой ультраметрической, т. е. кроме стандартного неравенства треугольника

$$
d_{p}(x, y) \leqslant d_{p}(x, z)+d_{p}(z, y), \quad x, y, z \in \mathbb{Q}_{p},
$$

она удовлетворяет сильному неравенству треугольника

$$
d_{p}(x, y) \leqslant \max \left[d_{p}(x, z), d_{p}(z, y)\right], \quad x, y, z \in \mathbb{Q}_{p} .
$$

Это следствие соответствующего неравенства для $p$-адической оценки

$$
|x+y|_{p} \leqslant \max \left[|x|_{p},|y|_{p}\right], \quad x, y \in \mathbb{Q}_{p} .
$$


Последняя доказывается непосредственно с помощью определения $p$-адической нормы.

Множество рациональных чисел $\mathbb{Q}$ вложено в $\mathbb{Q}_{p}$. Все арифметические операции (сложение, вычитание, умножение и деление) распространяются по непрерывности с $\mathbb{Q}$ на $\mathbb{Q}_{p}$, превращая последнее в топологическое поле, т. е. все арифметические операции на $\mathbb{Q}_{p}$ являются непрерывными. Заметим, что возможность определить деление является следствием использования только простых чисел $p>1$ в представленной конструкции. В принципе, можно продолжить работу с произвольно натуральными числами $p>1$ и получить топологическое кольцо, т. е. алгебраическую структуру, на которой все приведенные выше арифметические операции (кроме деления) хорошо определены и являются непрерывными.

В частности, поле $p$-адических чисел $\mathbb{Q}_{p}$ является локально компактной группой по отношению к операции сложения. Следовательно, существует мера Харара $d x$ на $\mathbb{Q}_{p}$. Эта мера является положительной и инвариантной относительно сдвигов, т. е. $d(x+a)=d x$, и нормируется с помощью соотношения $\int_{B_{1}(0)} d x=1$.

Функция $f: \mathbb{Q}_{p} \rightarrow \mathbb{C}$ является локально-постоянной, если для каждой точки $x$ существует шар с ненулевым радиусом, где $f$ является константой. В $p$-адическом случае локально-постоянные функции являются непрерывными. В некотором смысле они играют ту же роль, что и гладкие функции в вещественном анализе.

Пусть $\mathcal{D}\left(\mathbb{Q}_{p}\right)$ - линейное пространство локально-постоянных $\mathbb{C}$-значных функций с компактным носителем. Это пространство называется пространством основных функций Брюа-Швариа. Это пространство является р-адическим аналогом пространства гладких (бесконечно дифференцируемых) функций с компактным носителем, определенных на вещественной прямой, $\mathcal{D}(\mathbb{R})$. Последнее играет фундаментальную роль в теории распределений на вещественной прямой. Таким же образом $\mathcal{D}\left(\mathbb{Q}_{p}\right)$ служит основой теории распределений на $\mathbb{Q}_{p}$.

Заметим, что мы рассматриваем функциональные пространства комплекснозначных или вещественнозначных функций $p$-адического аргумента. Это особая часть классического гармонического анализа на локально-компактных группах; особенность связана с дополнительными алгебраическими структурами умножением и делением на $\mathbb{Q}_{p}$. Во многих применениях использовались функции $p$-адического аргумента, принимающие значения в $\mathbb{Q}_{p}$ или в одном из его алгебраических расширений, например в одном из квадратичных расширений - аналогов поля комплексных чисел $\mathbb{C}$ (см. [10]), включая соответствующую теорию распределений.

Теперь мы обратимся к конструкции теории распределений, основанной на пространстве пробных функций $\mathcal{D}\left(\mathbb{Q}_{p}\right)$. Это пространство может быть наделено естественной топологией индуктивного предела. Обозначим через $\mathcal{D}^{\prime}\left(\mathbb{Q}_{p}\right)$ множество всех непрерывных линейных функционалов, распределений Брюа-Швариа, на $\mathcal{D}\left(\mathbb{Q}_{p}\right)$.

Для пробной функции $\varphi \in \mathcal{D}\left(\mathbb{Q}_{p}\right)$ ее преобразование Фуръе определяется следующим образом:

$$
\widehat{\varphi}(\xi)=F[\varphi](\xi)=\int_{\mathbb{Q}_{p}} \varphi(x) e^{2 \pi i\{x \xi\}_{p}} d x .
$$

Оператор преобразования Фурье $F: \mathcal{D}\left(\mathbb{Q}_{p}\right) \rightarrow \mathcal{D}\left(\mathbb{Q}_{p}\right)$ является топологическим изоморфизмом.

Как и в стандартной теории распределений, преобразование Фурье в пространстве $\mathcal{D}^{\prime}\left(\mathbb{Q}_{p}\right)$ определяется как оператор, сопряженный с оператором преобразования 
Фурье в пространстве пробных функций $\mathcal{D}\left(\mathbb{Q}_{p}\right)$. Таким образом, для каждой пробной функции $\varphi \in \mathcal{D}\left(\mathbb{Q}_{p}\right)$

$$
\langle F[f], \varphi\rangle=\langle f, F[\varphi]\rangle .
$$

$p$-Адический дробный дифференциальный оператор $D^{\alpha}$ (для $\alpha>0$ ), известный в физике как оператор Владимирова ${ }^{2)}$, определяется с помощью следующего равенства:

$$
D^{\alpha} \varphi(x)=\int_{\mathbb{Q}_{p}}|\xi|_{p}^{\alpha} e^{-2 \pi i\{\xi x\}_{p}} \widehat{\varphi}(\xi) d \xi, \quad \xi \in \mathbb{Q}_{p} .
$$

Оператор $D^{\alpha}$ может быть представлен как нелокальный интегральный оператор. Мы также подчеркиваем, что, как было доказано в [10], нельзя определить локальный дифференциальный оператор (имеющий характерные черты обыкновенных дифференциальных операторов).

Как было замечено, оператор Владимирова широко использовался в математической и теоретической физике, особенно в теории комплексных неупорядоченных систем. Недавно его начали применять в некоторых задачах геофизики [5].

\section{3. p-АДИЧЕСКИЕ ВСПЛЕСКИ: СИСТЕМА КОЗЫРЕВА}

Мы применяем формализм р-адических всплесков в разделах 5,6 . Новичок в $p$-адике может, в принципе, обратиться к концептуальному разделу 4 и затем вновь вернуться в настоящий раздел или продолжить работу без чтения этого раздела и просто узнать, что в случае $p$-адики существует уже хорошо разработанная теория всплесков. Эта теория подобна теории всплесков на вещественной прямой. Вместо характеристических функций отрезков, как в случае вещественных всплесков Хаара, в $p$-адическом случае используются характеристические функции шаров. Основной отличительной чертой базиса p-адических всплесков является то, что дробные дифференциальные операторы (операторы Владимирова) диагонализируются в этом базисе. Это делает аппарат всплесков еще более полезным для анализа дробных дифференциальных уравнений, чем соответствующий аппарат с вещественными всплесками.

Пространство функций $\phi: \mathbb{Q}_{p} \rightarrow \mathbb{C}$, которые являются квадратично-интегрируемыми относительно меры Хаара, обозначается символом $L^{2}\left(\mathbb{Q}_{p}\right)$.

Следуя пионерской работе Козырева [1], мы вводим следующую систему p-адических всплесков (аналог системы Хаара на вещественной прямой):

$$
\psi_{q ; m n}(x)=p^{-m / 2} e^{2 \pi i\left\{q\left(p^{m} x-n\right) / p\right\}_{p}} \Omega\left(\left|p^{m} x-n\right|_{p}\right), \quad x \in \mathbb{Q}_{p},
$$

где $q=1,2, \ldots, p-1, m=0, \pm 1, \pm 2, \ldots$, a $n$ имеет вид $n=\sum_{s=j}^{-1} \alpha_{s} p^{s}$, где $j$ является отрицательным целым числом и $\alpha_{s}=0, \ldots, p-1$. Локалъное окно представлено математически с помощью функции $\Omega(\cdot)$ - характеристической функции $[0,1] \subset \mathbb{R}$. Оконная функция $\Omega\left(|\cdot|_{p}\right)$ является характеристической функцией единичного шара с центром в нуле, $B_{1}(0)$.

Важно заметить, что система $p$-адических всплесков $\left(\psi_{q ; m n}(x)\right)$ является ортонормированным базисом в $L^{2}\left(\mathbb{Q}_{p}\right)$.

\footnotetext{
2) Василий Сергеевич Владимиров был одним из основателей $p$-адической физики. Он активно использовал этот оператор в теории струн и в $p$-адических квантовых моделях (см., например, монографию [9]).
} 
Как отмечалось выше, система $p$-адических всплесков диагонализирует оператор дробного дифференцирования $D^{\alpha}$ (оператор Владимирова):

$$
D^{\alpha} \psi_{q ; m n}(x)=p^{\alpha(1-m)} \psi_{q ; m n}(x) .
$$

Базис всплесков Козырева, введенный в этом разделе, является естественным аналогом базиса всплесков Хаара. Общая теория $p$-адических всплесков была представлена в монографии [3] (см. также [34]).

\section{4. p-АДИЧЕСКАЯ СИСТЕМА КООРДИНАТ ДЛЯ СЕТЕЙ КАПИЛЛЯРОВ И УРАВНЕНИЯ БАЛАНСА ДЛЯ ПЛОТНОСТЕЙ ЖИДКОСТЕЙ}

Везде ниже переменная $x$ принадлежит полю $p$-адических чисел и временна́я переменная $t$ вещественна. Здесь $x$ является координатой сети капилляров в пористой среде, каждая траектория капилляров в пористой среде выражена с помощью ветви $p$-адического дерева. Центр этого дерева выбирается как произвольная точка сети капилляров в пористой среде, он играет роль центра координат, т. е. это чисто математический объект; мы не приписываем такому центру никакого геологического смысла. Таким образом, приписывая $p$-адическое число $x$ системе, мы знаем, в какой траектории капилляров она находится, и ничего больше. Таким образом, p-адическая модель дает схематическое представление сетей капилляров в пористой среде. В частности, размер капилляров не включен в геометрию. (Он может быть введен в модель с помощью коэффициентов уравнения аномальной реакции-диффузии; см., например, раздел 5.)

Исходя из динамики ${ }^{3)}$ мы находим концентрацию жидкости (нефти, воды или водомасляной эмульсии) в капиллярах. Однако мы не можем получить концентрацию жидкости в точно фиксированной точке евклидова физического пространства.

Мы также заметим, что каждый $p$-адический шар представляет пучок капиллярных траекторий: чем длиннее основание такого пучка, тем меньше радиус шара.

Рассмотрим жидкость, движущуюся в сети капилляров; обозначим ее концентрацию в капиллярах через $\rho(x, t)$. Динамика $\rho(x, t)$ описывается задачей Коши для следующего основного уравнения:

$$
\begin{gathered}
\frac{\partial \rho}{\partial t}(x, t)=\int_{\mathbb{Q}_{p}}[v(x \mid y) \rho(y, t)-v(y \mid x) \rho(x, t)] d y, \\
\rho(x, 0)=\rho_{0}(x),
\end{gathered}
$$

где $v(x \mid y)$ - вероятность перехода (в единицу времени) жидкости из капилляра $y$ в капилляр $x$.

Если количество жидкости в рассматриваемой древоподобной сети капилляров сохраняется, то должен выполняться закон сохранения

$$
\int_{\mathbb{Q}_{p}} \rho(x, t) d x=\text { const }=\int_{\mathbb{Q}_{p}} \rho_{0}(x) d x .
$$

\footnotetext{
3) Напомним, что динамика является вероятностной.
} 
Это эквивалентно условию

$$
0=\frac{\partial}{\partial t} \int_{\mathbb{Q}_{p}} \rho(x, t) d x=\int_{\mathbb{Q}_{p}} d x \int_{\mathbb{Q}_{p}}[v(x \mid y) \rho(y, t)-v(y \mid x) \rho(x, t)] d y .
$$

И интеграл в правой части равен нулю.

Если вероятность перехода является симметричной,

$$
v(x \mid y)=v(y \mid x)
$$

то динамика существенно проще. Заметим, что для динамики жидкости в сети капилляров эта симметрия вероятностей переходов является естественной. Также естественно считать, что вероятность перехода возрастает с уменьшением $p$-адического расстояния между двумя капиллярами. Самая простая математическая модель такого поведения задается вероятностями переходов, зависящими от расстояния,

$$
v(x \mid y)=v\left(|x-y|_{p}\right) .
$$

При моделировании динамики на сложных энергетических ландшафтах считается, что вероятность переходов определяется потенциалом типа Аррениуса, т. е.

$$
v\left(|x-y|_{p}\right)=\frac{A(T)}{|x-y|_{p}} \exp \left\{\frac{U\left(|x-y|_{p}\right)}{k T}\right\} .
$$

Наиболее часто используется вероятность переходов ${ }^{4)}$

$$
v\left(|x-y|_{p}\right)=\frac{1}{|x-y|_{p}^{\alpha+1}}
$$

Он приводит к дробным дифференциальным операторам Владимирова. В этом случае мы получаем $p$-адическую дробную диффузию:

$$
\frac{\partial \rho}{\partial t}(x, t)=D^{\alpha} \rho(x, t), \quad \rho(x, 0)=\rho_{0}(x) .
$$

Однако, как указывалось в разделе 1, в геофизических применениях нет причин ограничивать вероятности переходов вероятностями, которые приводят к операторам Владимирова. Таким образом, надо разработать теорию для произвольной динамики следующего типа:

$$
\frac{\partial \rho}{\partial t}(x, t)=\int_{\mathbb{Q}_{p}} v\left(|x-y|_{p}\right)[\rho(y, t)-\rho(x, t)] d y, \quad \rho(x, 0)=\rho_{0}(x)
$$

(см. соответствующие математические результаты в работах [23], [3], [4], [25], [26]).

Теперь предположим, что имеются несколько типов жидкости в разных состояниях; они обозначены с помощью индекса $i=1,2, \ldots, N-1$ (здесь мы делаем различие между, например, свободной нефтью и нефтью, связанной с поверхностью капилляров, а также между свободной и связанной водой). Обозначим их концентрации

\footnotetext{
4) Она может быть получена с помощью выбора соответствующего энергетического потенциала $U(x)$.
} 
в капиллярах через $\rho_{i}(x, t)$. Соответствующее основное уравнение имеет вид

$$
\begin{gathered}
\frac{\partial \rho_{i}}{\partial t}(x, t)=\sum_{j \neq i}\left(k_{i j}(x) \rho_{j}(x, t)-k_{j i} \rho_{i}(x, t)\right)+\int_{\mathbb{Q}_{p}} v_{i}\left(|x-y|_{p}\right)\left[\rho_{i}(y, t)-\rho_{i}(x, t)\right] d y \\
\rho_{i}(x, 0)=\rho_{0 i}(x) .
\end{gathered}
$$

Некоторые из коэфбициентов переноса $k_{i j}$ равны нулю; строго положительные коэффициенты перехода соответствуют нетривиальным переходам. Например, свободная нефть может быть захвачена поверхностью капилляров, и нефть, связанная с поверхностью капилляров, может покинуть поверхность, двигаясь сквозь капилляр.

Как и выше, мы можем с легкостью получить следующий простой, но важный (для обоснования модели) результат.

ПРЕДЛОЖЕНИЕ 1. Из уравнений (12) следует закон сохранения полного числа "частии":

$$
\int_{\mathbb{Q}_{p}} \sum_{i} \rho_{i}(x, t) d(x)=\text { const }=\int_{\mathbb{Q}_{p}} \sum_{i} \rho_{0 i}(x) d x .
$$

Перечислим математические задачи, связанные с системой основных уравнений:

1) существование и единственность решения в классе "гладких" функций, например в пространстве Лизоркина ${ }^{5)}$;

2) аналитические формулы для решений (например, в виде рядов всплесков);

3) стационарные решения и аналитические формулы для них.

Для наших целей стационарные решения и особенно их капиллярные носители являются особенно интересными. Рассмотрим динамику нефти в случайной пористой среде.

Сначала рассмотрим "чистую нефть", т. е. без водяной компоненты. Обозначим концентрацию нефти в капиллярах символом $\rho_{1}(x, t)$. Эта нефть не связана с твердыми поверхностями. Концентрация нефти, которая связана с поверхностью капилляров, обозначается символом $\rho_{2}(x, t)$. В нашей модели нефть течет в капиллярах, затем она может оказаться связанной с поверхностью капилляров и оставаться связанной некоторое время (нефть, связанная с твердым телом), а затем течь дальше и т. Д.

Таким же образом мы можем моделировать капиллярную динамику водомасляных эмульсий. Геологически эта динамика означает концентрацию капелек нефти в подпучке сети капилляров в случайных пористых средах. В терминах евклидовой геометрии это не что иное, как описание процесса образования кластера капилляров, где нефтяные капельки концентрируются и растут в размерах, образуя шаг за шагом нефтяной микрорезервуар.

Такой нефтяной резервуар может создаваться не только в природе, в процессе геологической эволюции, но также "искусственно" как часть программы по добыче нефти и особенно при выборе ускоренных методов добычи нефти, где стимулируется поток жидкости из твердых матриц. С помощью соответствующим образом выбранных давлений (которые определяют вероятности переходов $v(x \mid y)$ ) можно сконцентрировать нефть в ограниченной подсетке капилляров.

\footnotetext{
${ }^{5)}$ Решения в пространстве распределений не так интересны, так как $\rho_{i}(x, t)$ имеет физический смысл плотностей жидкостей.
} 


\section{5. ДИНАМИКА НЕФТИ: СВОБОДНОЙ И СВЯЗАННОЙ С ПОВЕРХНОСТЬЮ КАПИЛЛЯРОВ}

Здесь мы кратко представим модель, предложенную в работе [5]. Обозначим концентрацию свободной жидкости в капиллярах через $\rho_{1}(x, t)$. Эта жидкость не связана с поверхностью капилляров. Концентрация жидкости, которая связана с поверхностью, обозначается через $\rho_{2}(x, t)$. Мы получаем следующие два уравнения баланса:

$$
\begin{aligned}
& \frac{\partial}{\partial t} \rho_{1}(x, t)=-\left[K_{1}(x)+D_{x}^{\lambda} e^{\beta U_{1}(x)}\right] \rho_{1}(x, t)+K_{2}(x) \rho_{2}(x, t), \\
& \frac{\partial}{\partial t} \rho_{2}(x, t)=K_{1}(x) \rho_{1}(x, t)-\left[K_{2}(x)+D_{x}^{\lambda} e^{\beta U_{2}(x)}\right] \rho_{2}(x, t),
\end{aligned}
$$

где $\lambda>0$ и $\beta>0$ - параметры модели, описывающие соответственно степень дробности диффузии и степень связи с потенциалами $U_{j}(x)$, коэффициент перехода $K_{1}(x)$ выражает скорость реакции поглощения жидкости поверхностью капилляров, коэффициент перехода $K_{2}(x)$ выражает скорость реакции высвобождения поглощенной жидкости, $U_{1}(x)$ - потенциальная функция внутри $x$-й поры, а $U_{2}(x)$ - потенциальная функция, описывающая связь жидкости и поверхности капилляров в $x$-й траектории капилляров.

Можно показать, что в случае некоторых ограничений на коэффициенты переходов и потенциалы эта система имеет стационарное решение, сконцентрированное в $p$-адическом шаре, радиус которого определяется коэффициентами этой системы уравнений [5].

\section{6. КВАЗИЛИНЕЙНАЯ ДИФФУЗИЯ}

Как указывалось в разделе 1, нелинейные эффекты играют важную роль в моделировании переноса жидкостей в пористых средах. Однако теория нелинейных $p$-адических уравнений диффузии до сих пор не разработана. Имеется единственный результат [34], который касается следующего уравнения квазилинейной диффузии:

$$
\frac{\partial \rho}{\partial t}(x, t)=D^{\alpha} \rho(x, t)+\rho(x, t) f^{2 m}(x, t), \quad \rho(x, 0)=\rho_{0}(x) .
$$

Разлагая $\rho(x, t)$ по базису всплесков:

$$
\rho(x, t)=\sum_{s, j, a} \Lambda_{s, j, a}(t) \psi_{s, j, a}(x),
$$

мы получаем бесконечную систему обыкновенных дифференциальных уравнений для коэффициентов всплесков:

$$
\frac{d \Lambda_{s, j, a}(t)}{d t}+p^{\alpha(1-j)}|s|_{p} \Lambda_{s, j, a}(t)+p^{-m j} \Lambda_{s, j, a}^{2 m+1}(t)=0 .
$$

Интегрируя (19), мы получаем

$$
\frac{\Lambda_{s, j, a}^{2 m}(t)}{p^{\alpha(1-j)}|s|_{p}+p^{-m j} \Lambda_{s, j, a}^{2 m}(t)}=E_{s, j, a} e^{-2 m p^{\alpha(1-j)} t},
$$


где $E_{s, j, a}$ - константы, которые могут быть найдены из разложения по базису всплесков начальной функции $\rho_{0}(x)$. Здесь ключевым моментом является то, что всплески являются собственными функциями оператора Владимирова $D^{\alpha}$ (см. (2)). Таким образом,

$$
\Lambda_{s, j, a}(t)=\frac{\left(E_{s, j, a} p^{\alpha(1-j)}|s|_{p}\right)^{1 / 2 m} e^{-2 m p^{\alpha(1-j)} t}}{\left(1-p^{-m j} E_{s, j, a} e^{-2 m p^{\alpha(1-j)} t}\right)^{1 / 2 m}} .
$$

Итак, мы можем найти аналитические выражения для всплесковых коэффициентов этого квазилинейного дробно-дифференциального уравнения диффузии. Этот результат (хотя и простой) стимулирует поиск аналитических решений, например с помощью р-адической теории всплесков, для более сложных нелинейных дробно-дифференциальных уравнений диффузии.

\section{Список литературы}

[1] С. В. Козырев, “Теория всплесков как p-адический спектральный анализ”, Изв. РАН. Сер. матем., 66:2 (2002), 149-158.

[2] С.В. Козырев, " $p$-Адические псевдодифференциальные операторы и $p$-адические всплески", ТМФ, 138:3 (2004), 383-394.

[3] S. Albeverio, A. Yu. Khrennikov, V. M. Shelkovich, Theory of P-adic Distributions: Linear and Nonlinear Models, London Mathematical Society Lecture Note Series, 370, Cambridge Univ. Press, Cambridge, 2010.

[4] S. Albeverio, A. Yu. Khrennikov, V. M. Shelkovich, "The Cauchy problems for evolutionary pseudo-differential equations over $p$-adic field and the wavelet theory", J. Math. Anal. Appl., 375:1 (2011), 82-98.

[5] A. Khrennikov, K. Oleschko, M. de Jesús Correa López, "Application of p-adic wavelets to model reaction-diffusion dynamics in random porous media", J. Fourier Anal. Appl., 22:4 (2015), 809-822.

[6] I. V. Volovich, "p-Adic string", Class. Quantum Grav., 4:4 (1987), L83-L87.

[7] И. В. Волович, "p-Адическое пространство-время и теория струн”, ТМФ, 71:3 (1987), 337-340.

[8] I. Ya. Aref'eva, B. G. Dragović, I. V. Volovich, "On the $p$-adic summability of the anharmonic oscillator", Phys. Lett. B, 200:4 (1988), 512-514.

[9] V.S. Vladimirov, I. V. Volovich, E. I. Zelenov, p-Adic Analysis and Mathematical Physics, Series on Soviet and East European Mathematics, 1, World Sci., Singapore, 1994.

[10] A. Khrennikov, $p$-Adic Valued Distributions in Mathematical Physics, Mathematics and Its Applications, 309, Kluwer, Dordrecht, 1994.

[11] V.A. Avetisov, A. H. Bikulov, S. V. Kozyrev, "Application of $p$-adic analysis to models of breaking of replica symmetry", J. Phys. A: Math. Gen., 32:50 (1999), 8785-8791.

[12] G. Parisi, N. Sourlas, "p-Adic numbers and replica symmetry breaking", Eur. Phys. J. B, 14:3 (2000), 535-542.

[13] V.A. Avetisov, A. Kh. Bikulov, S. V. Kozyrev, V. A. Osipov, "p-Adic models of ultrametric diffusion constrained by hierarchical energy landscapes", J. Phys. A: Math. Gen., 35:2 (2002), 177-189.

[14] С. В. Козырев, "Ультраметрическая динамика как модель межбассейновой кинетики", Изв. НАН Армении. Матем., 41:5 (2006), 28-38.

[15] S. V. Kozyrev, "Ultrametric analysis and interbasin kinetics", p-Adic Mathematical Physics (Belgrade (Serbia) and Montenegro, 15-21 September, 2005), AIP Conference Proceedings, 826, eds. A. Yu. Khrennikov, Z. Rakic, I. V. Volovich, AIP, Melville, New York, 2006, 121-128. 
[16] S. Albeverio, R. Cianci, A. Yu. Khrennikov, " $p$-Adic valued quantization", $p$-Adic Numbers Ultrametric Anal. Appl., 1:2 (2009), 91-104.

[17] S. V. Kozyrev, "Dynamics on rugged landscapes of energy and ultrametric diffusion", $p$-Adic Numbers Ultrametric Anal. Appl., 2:2 (2010), 122-132.

[18] А.Ю. Хренников, Моделирование прочессов мышления в р-адических системах координат, Физматлит, М., 2004.

[19] D. Ben-Avraham, S. Havlin, Diffusion and Reactions in Fractals and Disordered Systems, Cambridge Univ. Press, Cambridge, 2000.

[20] B. O'Shaughnessy, I. Procaccia, "Diffusion on fractals", Phys. Rev. A, 32:5 (1985), 3073-3083.

[21] B. Berkowitz, R. P. Ewing, "Percolation theory and network modeling applications in soil physics", Surv. Geophys., 19:1 (1998), 23-72.

[22] J.-W. Kim, E. Perfect, H. Choi, "Anomalous diffusion in two-dimensional Euclidean and prefractal geometrical models of heterogeneous porous media", Water Resour. Res., 43 (2006), W01405.

[23] A. N. Kochubei, Pseudo-Differential Equations and Stochastics over Non-Archimedean Fields, Monographs and Textbooks in Pure and Applied Mathematics, 244, New York, 2001.

[24] A. N. Kochubei, "Radial solutions of non-Archimedean pseudo-differential equations", Pacific J. Math., 269:2 (2014), 355-369, arXiv: 1302.4850.

[25] W. A. Zúñiga-Galindo, "Fundamental solutions of pseudo-differential operators over $p$-adic fields", Rend. Sem. Mat. Univ. Padova, 109 (2003), 241-245.

[26] W.A. Zúñiga-Galindo, "Parabolic equations and Markov processes over $p$-adic fields,", Potential Anal., 28:2 (2008), 185-200.

[27] R. Metzler, J. Klafter, "The random walk's guide to anomalous diffusion: A fractional dynamics approach", Phys. Rep., 339:1 (2000), 1-77.

[28] R. Metzler, J. Klafter, "The restaurant at the end of the random walk: recent developments in the description of anomalous transport by fractional dynamics", J. Phys. A: Math. Gen., 37:31 (2004), R161-R208.

[29] S. Fedotov, S. H. Kim, H. Pitsch, "Anomalous Knudsen diffusion and reactions in disordered porous media", Center for Turbulence Research Annual Research Briefs, 323-331 (2007).

[30] B. I. Henry, T. A. M. Langlands, S. L. Wearne, "Anomalous diffusion with linear reaction dynamics: from continuous time random walks to fractional reaction-diffusion equations", Phys. Rev. E, 74:3 (2006), 031116, 15 pp.

[31] K. Malek, M.-O. Coppens, "Knudsen self- and Fickian diffusion in rough nanoporous media", J. Chem. Phys., 119:5 (2003), 2801-2811.

[32] A. J. Dammers, M.-O. Coppens, "Anomalous Knudsen diffusion in simple pore models", Diff. Fund., 2 (2005), 14.1-14.2.

[33] A. N. Kochubei, "Distributed order calculus and equations of ultraslow diffusion", J. Math. Anal. Appl., 340:1 (2008), 252-281.

[34] A. Yu. Khrennikov, V. M. Shelkovich, "Non-Haar $p$-adic wavelets and their application to pseudo-differential operators and equations", Appl. Comput. Harmon. Anal., 28:1 (2010), $1-23$.

[35] K. Oleschko, J.-F. Parrot, G. Ronquillo, S. Shoba, G. Stoops, V. Marcelino, "Weathering: Toward a fractal quantifying", Math. Geology, 36:5 (2004), 607-627.

[36] K. Oleschko, G. Korvin, B. Figueroa, M. A. Vuelvas, A. S. Balankin, L. Flores, D. Carreón, "Fractal radar scattering from soil", Phys. Rev. E, 67:4 (2003), 041403; Erratum, 68:3 (2003), 039904, 1 pp. 